\title{
Personal LocationMessaging
}

\author{
Markku Saarelainen \\ Oy Arbonaut Ltd, Torikatu 21 c 80100 Joensuu, Finland. www.arbonautcom, \\ markku.saarelainen@arbonaut.com
}

\begin{abstract}
When communicating with other people, we are normally aware of our surroundings, as a context of our communication. Geographical representation as a part of messaging has a long history in traditional media. For example, we send postcards with a picture and a postage stamp. The LocationMessaging Server is a messaging platform that provides the possibility to utilize location information to enrich mobile and web based messaging. On one hand, the Web interface of the Server represents a possibility to store, create, organize and deliver messages that include a location reference. A wide range of map material can be used to visualize the location a message created or received contains. On the other hand, mobility gives the freedom to create and receive geographically enriched messages anywhere; LocationMessaging is used to store your own Points of Interest, to inform others about your location, to search and find places and people.
\end{abstract}

\section{Introduction}

The LocationMessaging Suite, FleetManager and MINDER form a services family that supports the LocationMessaging needs of different usergroups. The market for location based mobile services is expected to grow to 14 billion euro by 2006 (Applied Business Intelligence). The LocationMessaging Suite is a general solution for mobile location messaging which allows the users to locate their friends or contacts. It is also possible to search the personal surroundings of your current location with keywords, and to find matching Points Of Interest. LocationMessaging is based on SMS messages or WAP, with amendments, which include:

- Geo-coded location documents

- Geo-coded location messages

- GeoSearch

For the user, the functionality is easy to adopt. The users can store and edit LocationMessages in a personal LocationNotebook, or in a public Open LocationNotebook. Incoming messages are stored in an Inbox, and all sent messages, whether sent through the Web or with a mobile terminal, are stored in an Outbox.

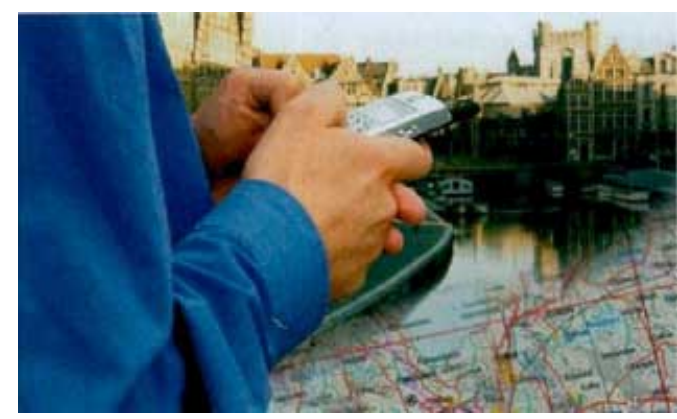

Fig. 1: LocationMessaging 
LocationMessaging allows simple answers to, for example, the following tasks:

- Replying to "Where R U?"

- Sharing "This place is cool!" with friends

- Agreeing to "Can we meet ... :-)"

- Checking "Did Suzie catch the bus?"

- Finding out what other people like you found interesting in this town The LocationMessaging Services have four common elements:

- LocationNotebook,

- Composer,

- ContactBook and the

- User Profile.

All of these components are available when using the LocationMessaging at the website or with a mobile phone.

LocationNotebook is a service that allows you to store LocationMessages in a database. LocationMessages, your personal Points of Interest, can be created on a website or on a mobile phone using SMS. LocationNotebook allows the user to create folders for the messages and to edit the messages afterwards to suit the needs of the user. This may involve, for example, selecting another icon or adding text to deepen the description of saved data.

Composer is a tool to create LocationMessages. Composing a message takes three steps:

1. selecting send or save functionality,

2. selecting location with referring icon, and

3. writing the text of the message.

At a LocationMessaging website, the Composer can be used with maps and Locationcoding to refer to the location indicated in the LocationMessage. The Locationcoding engine is very useful when an address needs to be converted into coordinates, and further on to a point on a map. Recipients can be selected from a ContactBook, or from a LocationMessaging specific contact database. Alternatively, the phonenumbers or e-mail addresses, respectively, of the recipients can be typed in.

ContactBook is a user defined contact database. By using the ContactBook, users can define the personal contact data, addresses, phone numbers, e-mail accounts and the preferred contact methods of the recipients of their LocationMessages. The ContactBook also manages the highly important setting of permissions for each Contact on if and when they are allowed to apply the friend finder function to the owner of the ContactBook. It is also possible to create Contact groups for sending group messages. Contact groups can include subgroups, and they can overlap partially with other Contact groups.

User Profile is a tool to control and modify each user's own messaging devices. A user can add, edit and priorize the devices by the context the messages are received. 
The web interface is used as a default, so that a LocationMessaging website automatically keeps a log of all LocationMessages sent and received, unless the user explicitly disables this function. In addition, the User Profile determines the default values for proximity search radius, emergency messages and for LocationNotebook mailboxes.

All of the messaging environments have common elements, which can be found as generic modules in the Suite or combined as applications, for example, the FleetManager is designed for asset-tracking use so it has additional components used in vehicle tracing whilst MINDER is a solution for personal usage supporting security and safety application such as monitoring the whereabouts of children.

\subsection{FleetManager}

FleetManager is a generic tool to locate vehicles, workforce and valuable assets - and to communicate with them. Additional parts depend strongly on customers need. Typically the implementation includes proximity search engine, interfacing with the customers point of interest databases and raster and vector map servers.

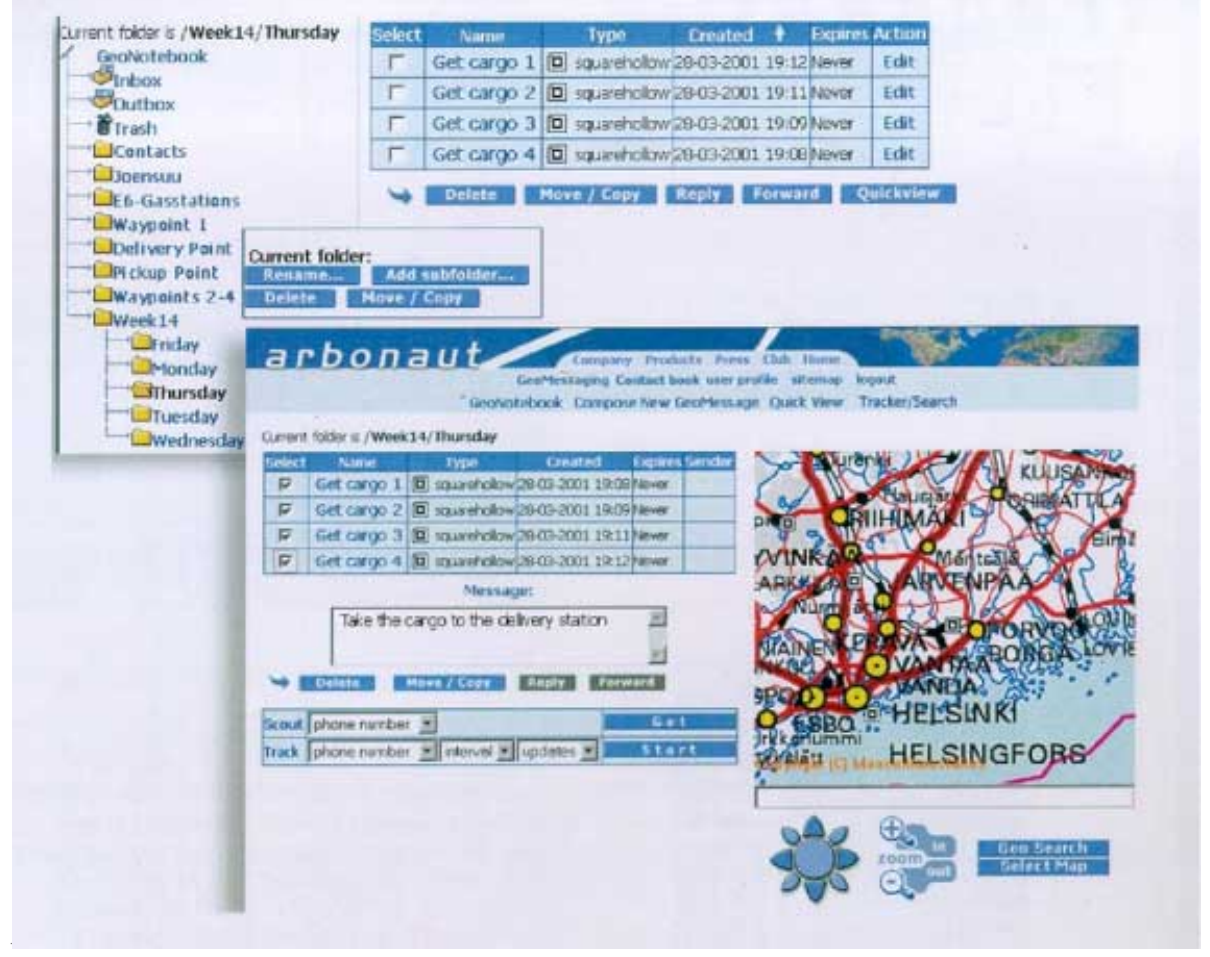

Fig. 2 FleetManager 


\subsection{MINDER}

MINDER (Movement INformation DElivered Regularly) is a version of FleetManager that has been customized for personal location tracking for example determining the location of a child. MINDER can be used to locate personnel outdoors, in urban areas and indoors.

\section{General features of application layer}

The features of the application layer are modular entities and can be integrated into the existing location information and/or messaging system of any application.

Operating system independency. The LocationMessaging-solutions can be flexibly transferred on to any device, operating system and application environments.

\section{Modules of application layer and their general description}

Messaging solutions. a two-way messaging system has been implemented, illustrated in Fig.3, between the Web and mobile terminals :

\begin{tabular}{|c|c|c|c|c|}
\hline & \multicolumn{4}{|c|}{ Sending } \\
\hline \multirow{5}{*}{ له } & & WEB & WAP & SMS \\
\hline & WEB & $\checkmark$ & 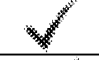 & $\checkmark$ \\
\hline & WAP & 4 & $\checkmark$ & 4 \\
\hline & SMS & $\checkmark$ & 4 & $V$ \\
\hline & Email & 4 & $\checkmark$ & $\checkmark$ \\
\hline
\end{tabular}

Fig.3. Cross Media LocationMessaging

Location information-based messages can be sent/received in/from the system and inspected on a map. GPS/GSM-devices can be located and the points in question can be displayed on a map, marked for example with a distinctive symbol. Scout- and Track-features can be used with GPS/GSM-terminals. Also so called Trigger-points, which work as automatic trigger areas of location information, can be sent to terminals

User groups. FleetManager and MINDER support several user groups within the application. Different user rights and views can be given to different kinds of users.

Positioning. The current version of the system supports terminal-specific GPSpositioning. Support for network positioning will be in use as soon as operator(s) 
present(s) a functioning and a comprehensive service in Finland. In addition, the system utilises Locationcoding in producing and using location information.

Map management. It is possible to use vector and raster map material in the FleetManager-and MINDER applications in three different ways.

- Customer uses an external map provider (e.g. Genimap or Novo) from whom the maps needed in the service can be obtained for use both in the Internet and in mobile devices

- Customer saves its own digital map material on the image server of Arbonaut, from which the maps needed in the service can be obtained for use for both in the Internet and in mobile devices.

- Arbonaut forms an interface to an existing map server of the user, from which the maps needed in the service can be taken into use for both in the Internet and in mobile devices.

It is possible to use all map materials in the Internet using a browser, which supports Locationcoded navigation, developed by Arbonaut. Maps can be downloaded also to PDA, WAP and Benefon ESC! devices.

User-specific databases. Each user/user group can save LocationMessages into a private database structure, which can be modified user/user group-specifically.

Terminal support. FleetManager and MINDER -applications support the following GPS/GSM devices:

- $\quad$ Benefon ESC! - mobile phone with map display

- Benefon Track - telematics phone for professional use

- Falcom - independent mobile positioning device (Asset tracker)

In addition, normal GSM-terminals and WAP and PDA devices (palmtop) can be connected to the applications.

\section{Architectural overview}

The LocationMessaging System extends standard Messaging Systems by incorporating a special treatment for Location Based Information. The LocationMessaging System provides:

- Management of personal information

- Access to Community or Public information

- Exchange of information between people

- Map Management to display Location Based Information.

The LocationMessaging System can be divided in the following components:

- LocationMessaging Server

- LocationMessengers

- LocationMessaging Services 
The LocationMessaging Server hosts the LocationMessaging Services and the Location Based Information depicted in Fig.4. LocationMessenger is the generic name for a messaging client of a LocationMessaging application. A LocationMessaging System includes other types of clients like Administration Tools. The LocationMessaging System includes interfaces to:

- Map servers through a Map Proxy

- Positioning Systems, especially network based positioning systems

- Traditional messaging systems (e-mail, www, fax, SMS, ...)

- External Content Providers and User Databases

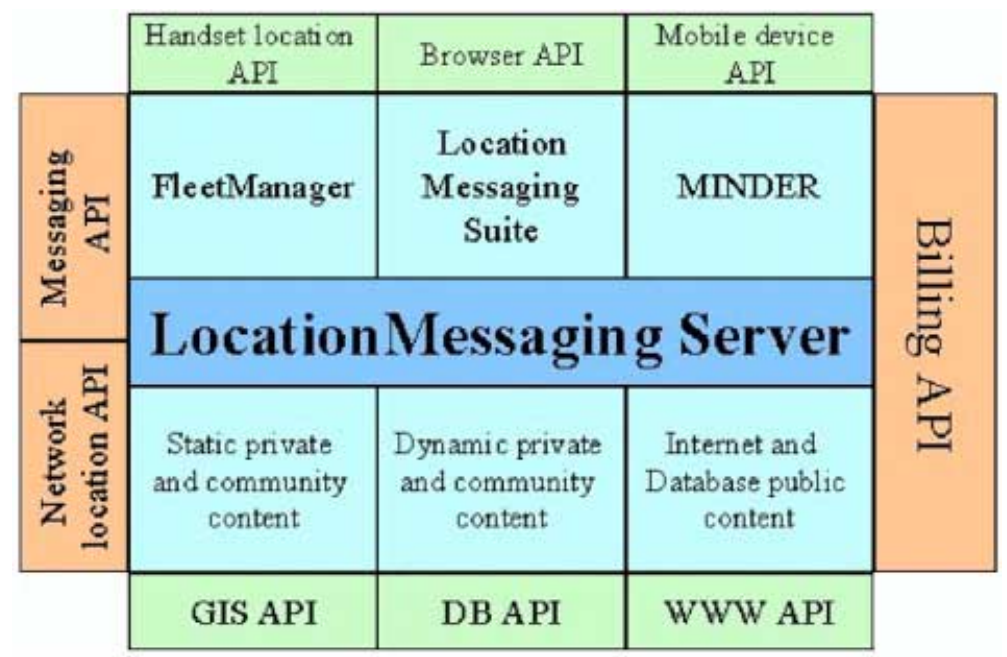

Fig. 4 LocationMessaging Server. Layered structure of the server consists of the core server, service layers (as the Suite, FleetManager and MINDER as well as the content layer) and the communication API and database API layers. In addition there are more customer dependent network location API, messaging API and billing API layers.

\section{Application integration interface}

The LocationMessaging Suite has been designed to implement and port into various technological environments. Through its adaptable interfaces the LocationMessaging Server can be tailored and harnessed to address the users needs. Typical API's for integration are:

- $\quad$ Existing user database

- Network positioning systems

- Billing interface

- GIS interfaces 


\section{Friend Finder and Child monitoring}

Mobile communication today is free from place, yet our daily actions and decisions are more often defined by our surroundings. Having the possibility to locate ourselves in relation to the services we want to consume and indeed to our social reference group, our friends, families, workgroups and people we share hobbies with gives us more added value and basis to make decisions and to communicate.

Friend finder, an application of MINDER, is a solution designed for every mobile and web user to locate themselves and their friends and to use this information to enrich their messaging. Based on the fact that the operators network positioning technology is being harnessed to answer each query made by the LocationMessaging Suite, the Friend finder falls into two appearances: the web and the mobile one.

The Friend finder at the Web

The subscribers can use their private user interface to communicate with their friends. The LocationMessaging system enables the user to make queries about their friend's location. The position information is being derived from the mobile operators network positioning server and the results are brought to the subscriber with indicating icon on a referring maps over the area. The easy usability of the service has been one of the key factors in Friend finder. With the -best practice in semantics- graphical user interface it is easy to select your friend or a group of friends from your contact book and choose suitable friend find option from the service. As a result the list of position query results will be displayed with referring locations.

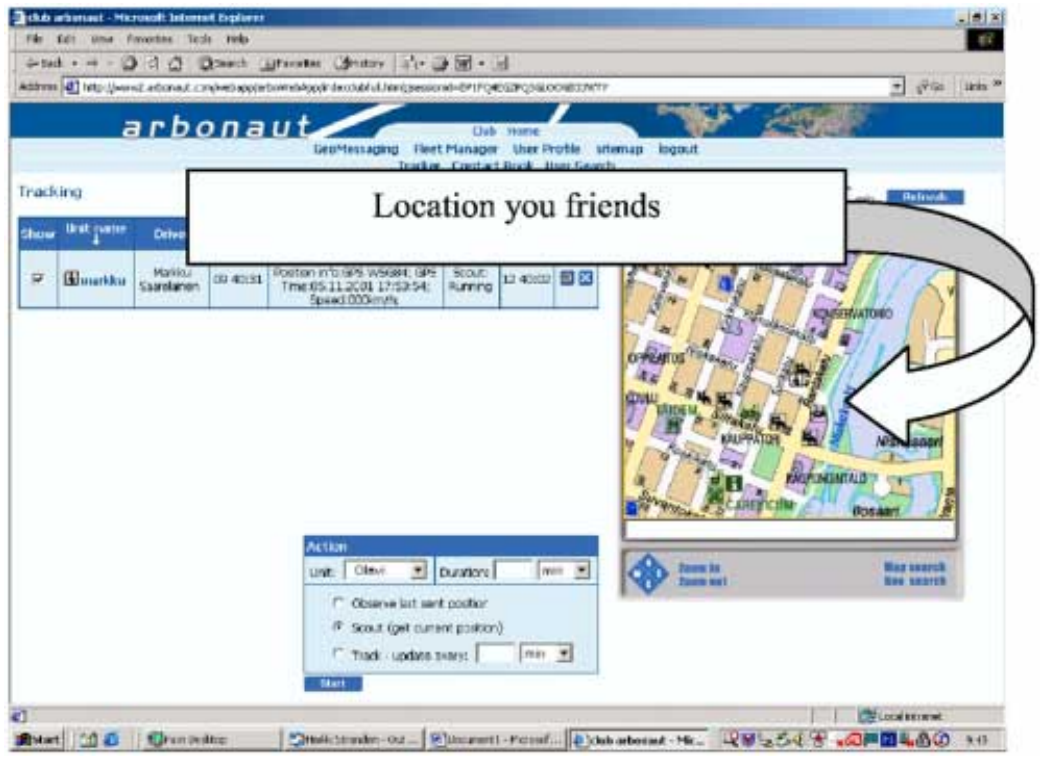

Fig. 5 Friend finder 
For each result - the position of the friend - it is possible to center the map according to that position and to see the status (the friend find profile) of the friend. By clicking the referring name of the friend at the result list it is possible to open a certain composer view with your friends contact info already filled in the recipient field and the location of the friend as a starting point to select desired position of your LocationMessage. The message can be sent as a web message, WAP, e-mail or by SMS.

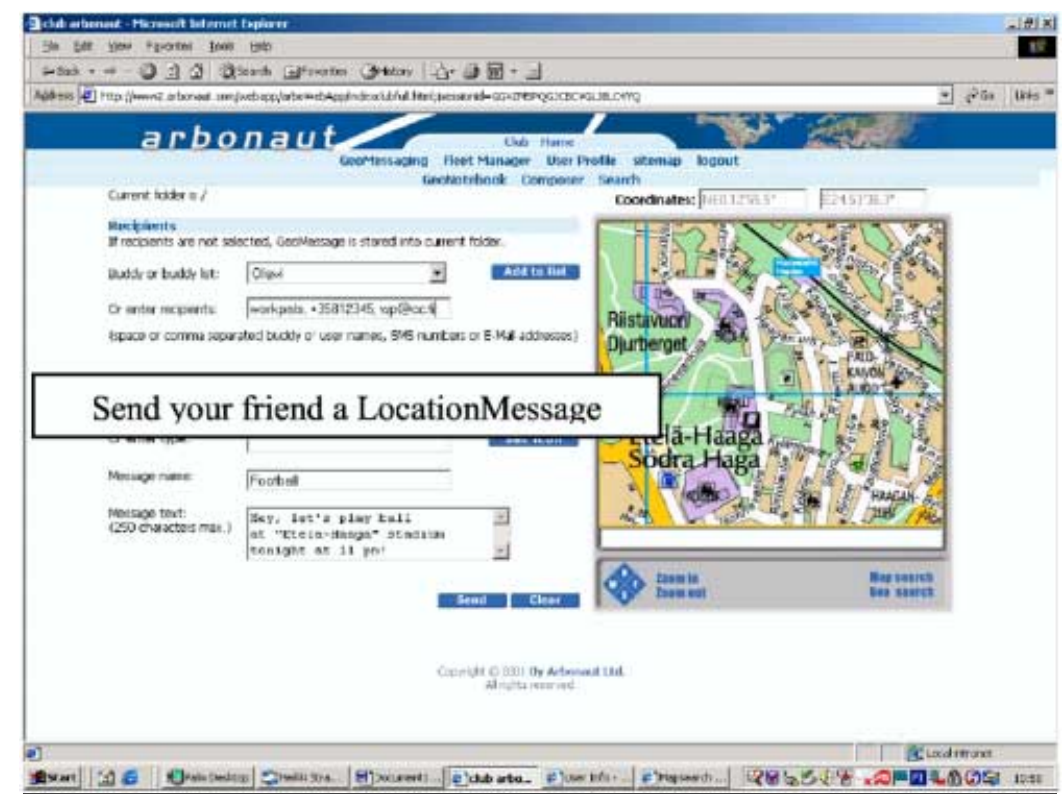

Fig. 6 Web-based LocationMessaging

Crossmedia LocationMessaging gives the possibility for both web and mobile users to communicate with location in two ways. Sending and receiving generally requires subscribing the services in domain, however the receiving the LocationMessage by SMS or email is available also for nonsubscribers

Friend find in mobile phones

Friend find can be carried out also by using the mobile phone. Here there are two different scenarios - the WAP and SMS. The WAP can be used in a similar way than the web. Especially the utilization of WAP-mapping technology the friend find results can be displayed in a terminal specific mode. SMS based friend find results are basically text: the direction and distance of the friends location can be displayed in addition to the nearest Point of Interest of the area 


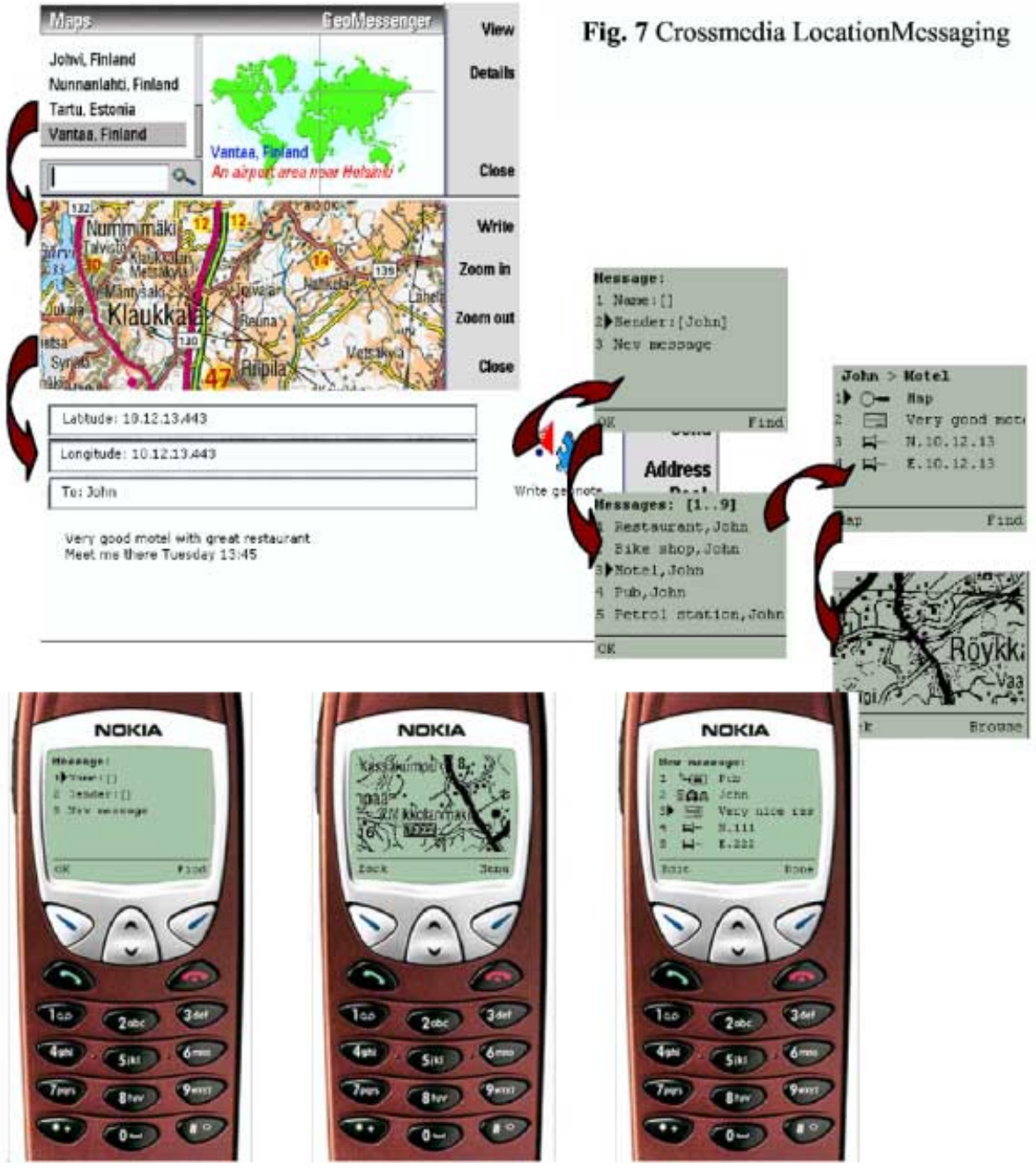

The LocationMessaging system can also be used to provide the foundations to develop a number of Mobile safety types of applications such as child monitoring as depicted in fig .8. MINDER can be used to monitor personnel outdoors (using GPS) in urban canyons (using network positioning) and even indoors.

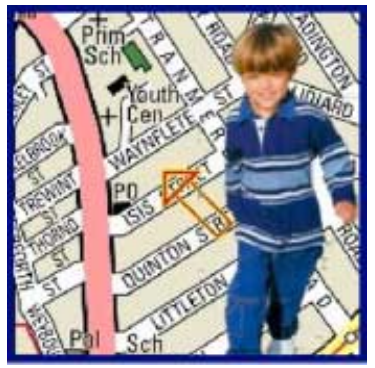

Fig. 8 Child monitoring using MINDER 